\title{
Existence results of second-order impulsive neutral functional differential inclusions in Banach spaces
}

\section{Taotao Li and Jianli Li*}

"Correspondence: ljianli@sina.com Department of Mathematics, Hunan Normal University, Changsha,

Hunan 410081, China

\begin{abstract}
In this paper, we investigate the existence of solutions for a class of second-order impulsive neutral functional differential inclusions in Banach spaces. Sufficient conditions for the existence are derived with the help of the fixed point theorem for multivalued maps due to Dhage.
\end{abstract}

Keywords: impulse neutral functional differential inclusions; second-order; existence; fixed point theorem

\section{Introduction}

In this paper, we shall study a class of initial value problems for second-order impulsive neutral functional differential inclusions in Banach spaces described in the form

$$
\left\{\begin{array}{l}
\frac{d}{d t}\left[y^{\prime}(t)-g\left(t, y_{t}\right)\right] \in F\left(t, y_{t}\right), \quad \text { a.e. } t \in J=[0, T], t \neq t_{k}, k=1, \ldots, t_{m}, \\
\left.\triangle y\right|_{t=t_{k}}=I_{k}\left(y\left(t_{k}^{-}\right)\right), \quad k=1, \ldots, m, \\
\left.\triangle y^{\prime}\right|_{t=t_{k}}=\bar{I}_{k}\left(y\left(t_{k}^{-}\right)\right), \quad k=1, \ldots, m, \\
y(t)=\phi(t), \quad t \in[-r, 0], \quad y^{\prime}(0)=\eta,
\end{array}\right.
$$

where $F: J \times \mathcal{D} \rightarrow P(E)$ is a multivalued map, $g: J \times \mathcal{D} \rightarrow E$ is a given function, $\mathcal{D}=$ $\{\psi:[-r, 0] \rightarrow E \mid \psi$ is continuous everywhere except for a finite number of points $s$ at which $\psi(s)$ and the right limit $\psi\left(s^{+}\right)$exist and $\left.\psi\left(s^{-}\right)=\psi(s)\right\}, \phi \in \mathcal{D}(0<r<\infty), P(E)$ is the family of all subsets of $E, 0=t_{0}<t_{1}<\cdots<t_{m}<t_{m+1}=T, I_{k}: E \rightarrow E(k=1,2, \ldots, m)$, $\bar{I}_{k}: \bar{E} \rightarrow E$, and $\eta \in E,\left.\Delta y\right|_{t=t_{k}}=y\left(t_{k}^{+}\right)-y\left(t_{k}^{-}\right) ; y\left(t_{k}^{-}\right)$and $y\left(t_{k}^{+}\right)$represent the left and right limits of $y(t), t=t_{k}$, respectively, and $E$ a real separable Banach space with norm $\|\cdot\|$.

For any continuous function $y$ defined on $[-r, T] \backslash\left\{t_{1}, t_{2}, \ldots, t_{m}\right\}$ and $t \in J$, we denote by $y_{t}$ the element of $\mathcal{D}$ defined by $y_{t}(\theta)=y(t+\theta), \theta \in[-r, 0]$. Here $y_{t}(\cdot)$ represents the history of the state from time $t-r$, up to the present time $t$.

In recent years, the theory of impulsive differential equations or inclusions has become an active area of investigation due to their applications in the fields of mechanics, electrical engineering, medicine biology, ecology, and so on. It has attracted great interest of researchers. For example, with the aid of Schaefer's theorem, an existence result for firstand second-order impulsive neutral functional differential equations in Banach spaces has 
been given by the authors in [1]. By means of a fixed point theorem for condensing multivalued map, solvability of impulsive neutral evolution differential inclusions with statedependent delay has been given by the authors in [2]. There are many other methods such as in $[3,4]$ that have been for various initial and boundary value problems for impulsive differential inclusions. However, recently much attention has been paid to using a fixed point theorem for multivalued maps due to Dhage to solve the problem for impulsive differential inclusions. One can refer to $[5,6]$ and the references therein. Motivated by the previous mentioned paper, we will study the existence of solutions for a class of second-order impulsive neutral functional differential inclusions in Banach spaces. Sufficient conditions for the existence are given by means of the fixed point theorem for multivalued maps due to Dhage [7]. For the IVP (1.1) we refer to [8].

\section{Preliminaries}

In this section, we shall introduce some basic definitions and lemmas which are used throughout this paper.

For $\psi \in \mathcal{D}$, the norm of $\psi$ is defined by $\|\psi\|_{\mathcal{D}}=\sup \{\|\psi(\theta)\|:-r \leq \theta \leq 0\}$.

$A C^{i}(J, E)$ is the space of $i$-times differentiable functions $y: J \rightarrow E$, whose $i$ th derivative, $y^{i}$, is absolutely continuous.

$\Omega:=\Omega([-r, T])=\left\{y:[-r, T] \rightarrow E: y_{k} \in C\left(J_{k}, E\right), k=0, \ldots, m\right.$ and there exist $y\left(t_{k}^{-}\right)$and $y\left(t_{k}^{+}\right), k=1, \ldots, m$, with $\left.y\left(t_{k}^{-}\right)=y\left(t_{k}\right)\right\}$, which is a Banach space with the norm

$$
\|y\|_{\Omega}=\sup \left\{\left\|y_{k}(t)\right\|: t \in[-r, T]\right\} .
$$

Let $P(X)$ denote the class of all nonempty subsets of $X$. Let $P_{\mathrm{bd}, \mathrm{cl}}(X), P_{\mathrm{cp}, \mathrm{cv}}(X), P_{\mathrm{bd}, \mathrm{cl}, \mathrm{cv}}(X)$, and $P_{\mathrm{cd}}(X)$ denote, respectively, the family of all nonempty bounded-closed, compactconvex, bounded-closed-convex and compact-acyclic (see [9]) subset of $X$. For $x \in X$ and $Y, Z \in P_{\mathrm{bd}, \mathrm{cl}}(X)$, we define $D(x, Y)=\inf \{\|x-y\|: y \in Y\}, \rho(Y, Z)=\sup _{a \in Y} D(a, Z)$, and the Hausdorff metric $H: P_{\mathrm{bd}, \mathrm{cl}}(X) \times P_{\mathrm{bd}, \mathrm{cl}}(X) \rightarrow R^{+}$by $H(A, B)=\max \{\rho(A, B), \rho(B, A)\}$.

$F$ is called upper semicontinuous (for brevity: u.s.c.) on $X$, if for each $x_{*} \in X$, the $F\left(x_{*}\right)$ is nonempty, closed subset of $X$, and if, for each open of $V$ of $X$ containing $F\left(x_{*}\right)$, there exists an open neighborhood $N$ of $x_{*}$ such that $F(N) \subseteq V$. F is said to be complete if $F(N)$ is relatively compact, for every bounded subset $V \subseteq X$.

If the multivalued map $F$ is completely continuous with nonempty compact values, then $F$ is u.s.c. if and only if $F$ has a closed graph (i.e. $x_{n} \rightarrow x_{*}, y_{n} \rightarrow y_{*}, y_{n} \in F\left(x_{n}\right)$ imply $y_{*} \in$ $F\left(x_{*}\right)$.

A point $x_{0} \in X$ is called a fixed point of the multivalued map $G$ if $x_{0} \in F\left(x_{0}\right)$. For more details of the multivalued maps, see the books of Deimling [10].

Definition 2.1 Let $F: X \rightarrow P_{\mathrm{bd}, \mathrm{cl}}(X)$ be a multivalued map. Then $F$ is called a multivalued contraction if there exists a constant $k \in(0,1)$ such that for each $x, y \in X$ we have

$$
H(F(x), F(y)) \leq k\|x-y\| .
$$

The constant $k$ is called a contraction constant of $F$.

Lemma 2.1 Let $E$ be a Banach space. Let $F: J \times E \rightarrow P_{\mathrm{cp}, \mathrm{cv}}(E)$ be an $L^{1}$-Carathéodory multivalued map with $S_{F, y}:=\left\{f \in L^{1}(J, E): f(t) \in F(t, y(t))\right.$ for a.e. $\left.t \in J\right\} \neq \varnothing$ and let $\Gamma$ be 
a linear continuous mapping from $L^{1}(J, E)$ to $C(J, E)$, then the operator $\Gamma \circ S_{F}: C(J, E) \rightarrow$ $P_{\mathrm{cp}, \mathrm{cv}}(C(J, E)), y \mapsto\left(\Gamma \circ S_{F}\right)(y):=\Gamma\left(S_{F, y}\right)$ is a closed graph operator in $C(J, E) \times C(J, E)$.

Theorem 2.1 Let E be a Banach space, $\Phi_{1}: E \rightarrow P_{\mathrm{bd}, \mathrm{cl}, \mathrm{cv}}(E)$ and $\Phi_{2}: E \rightarrow P_{\mathrm{cp}, \mathrm{cv}}(E)$ be two multivalued maps satisfying:

(i) $\Phi_{1}$ is a contraction with a contraction constant $k$, and

(ii) $\Phi_{2}$ is completely continuous.

Then either

(1) the operator inclusion $x \in \Phi_{1} x+\Phi_{2} x$ has a solution, or

(2) the set $G=\left\{x \in E: x \in \lambda \Phi_{1} x+\lambda \Phi_{2} x\right\}$ is unbounded for $\lambda \in(0,1)$.

Definition 2.2 The multivalued map $F: J \times E \rightarrow P(E)$ is said to be $L^{1}$-Carathéodory if

(i) $t \mapsto F(t, u)$ is measurable for each $u \in E$;

(ii) $u \mapsto F(t, u)$ is upper semicontinuous on $E$ for all $t \in J$;

(iii) for each $\rho>0$, there exists $\varphi_{\rho} \in L^{1}\left(J, R^{+}\right)$such that

$$
\|F(t, u)\|_{P(E)}=\sup \{|v|: v \in F(t, u)\} \leq \varphi_{\rho}(t), \quad \forall\|u\| \leq \rho \text { and for a.e. } t \in J .
$$

\section{Main result}

Definition 3.1 A function $y \in \Omega \cap A C^{1}\left(\left(t_{k}, t_{k+1}\right), E\right), k=0, \ldots, m$, is said to be a solution of (1.1) if $y$ satisfies the differential inclusion $\frac{d}{d t}\left[y^{\prime}(t)-g\left(t, y_{t}\right)\right] \in F\left(t, y_{t}\right)$ a.e. on $J\left\{t_{1}, t_{2}, \ldots, t_{m}\right\}$, the conditions $\left.\Delta y\right|_{t=t_{k}}=I_{k}\left(y\left(t_{k}^{-}\right)\right),\left.\Delta y^{\prime}\right|_{t=t_{k}}=\bar{I}_{k}\left(y\left(t_{k}^{-}\right)\right), k=1, \ldots, m, y(t)=\phi(t), t \in[-r, 0]$, and $y^{\prime}(0)=\eta$.

\section{Theorem 3.1 Assume the following.}

$\left(\mathrm{H}_{1}\right) \quad\|g(t, u)-g(t, \bar{u})\| \leq p\|u-\bar{u}\|$, for each $u, \bar{u} \in \mathcal{D}$, where $p$ is a nonnegative constant.

$\left(\mathrm{H}_{2}\right)\left\|I_{k}(y)-I_{k}(\bar{y})\right\| \leq c_{k}\|y-\bar{y}\|$, for each $y, \bar{y} \in E, k=1, \ldots, m$, where $c_{k}$ are nonnegative constants, and there exist constants $c_{k}^{\prime}$ such that $\left|I_{k}(y)\right| \leq c_{k}^{\prime}, k=1, \ldots, m$, for each $y \in E$.

$\left(\mathrm{H}_{3}\right)\left\|\bar{I}_{k}(y)-\bar{I}_{k}(\bar{y})\right\| \leq d_{k}\|y-\bar{y}\|$, for each $y, \bar{y} \in E, k=1, \ldots, m$, where $d_{k}$ are nonnegative constants, and there exist constants $d_{k}^{\prime}$ such that $\left|\bar{I}_{k}(y)\right| \leq d_{k}^{\prime}, k=1, \ldots, m$, for each $y \in E$.

$\left(\mathrm{H}_{4}\right)$ The function $g$ is completely continuous and there exist constants $0 \leq c_{1}^{*} \leq 1$ and $c_{2}^{*} \geq 0$ such that $|g(t, u)| \leq c_{1}^{*}\|u\|+c_{2}^{*}, t \in J, u \in \mathcal{D}$ are satisfied.

$\left(\mathrm{H}_{5}\right) F: J \times \mathcal{D} \rightarrow P_{\mathrm{b}, \mathrm{cp}, \mathrm{cv}}(E)$ is an $L^{1}$-Carathéodory function.

$\left(\mathrm{H}_{6}\right)\|F(t, u)\| \leq p_{1}(t) \psi\left(\|u\|_{\mathcal{D}}\right)$ for almost all $t \in J$ and all $u \in \mathcal{D}$, where $p_{1} \in L^{1}\left(J, R_{+}\right)$and $\psi: R_{+} \rightarrow(0, \infty)$ is continuous and increasing with

$$
\int_{0}^{T} M(s) d s<\int_{\bar{c}}^{\infty} \frac{d s}{s+\psi(s)}
$$

where $\bar{c}=\|\phi\|_{\mathcal{D}}+\left[\|\eta\|+c_{1}^{*}\|\phi\|_{\mathcal{D}}+2 c_{2}^{*}\right] T+\sum_{k=1}^{m}\left[c_{k}^{\prime}+\left(T-t_{k}\right) d_{k}^{\prime}\right]$ and $M(t)=$ $\max \left\{c_{1}^{*}, p_{1}(t)\right\}$.

If

$$
p T+\sum_{k=1}^{m}\left(c_{k}+\left(T-t_{k}\right) d_{k}\right)<1
$$

then the IVP (1.1) has at least one solution on $[-r, T]$. 
Proof Consider the operator $N: \Omega \rightarrow P(\Omega)$ defined by

$$
N(y)= \begin{cases}\phi(t), & t \in[-r, 0], \\ \phi(0)+[\eta-g(0, \phi(0))] t+\int_{0}^{t} g\left(s, y_{s}\right) d s+\int_{0}^{t} \int_{0}^{s} v(u) d u d s & \\ \quad+\sum_{0<t_{k}<t}\left[I_{k}\left(y\left(t_{k}\right)\right)+\left(t-t_{k}\right) \bar{I}_{k}\left(y\left(t_{k}\right)\right)\right], & t \in J,\end{cases}
$$

where $v \in S_{F, y}=\left\{y \in L^{1}(J, E): v(t) \in F\left(t, y_{t}\right)\right.$ for a.e. $\left.t \in J\right\}$. It is clear that the fixed points of $N$ are solutions of problem (1.1).

Let

$$
\begin{aligned}
N_{1}(y)= & {[\eta-g(0, \phi(0))] t+\int_{0}^{t} g\left(s, y_{s}\right) d s } \\
& +\sum_{0<t_{k}<t}\left[I_{k}\left(y\left(t_{k}\right)\right)+\left(t-t_{k}\right) \bar{I}_{k}\left(y\left(t_{k}\right)\right)\right]
\end{aligned}
$$

and

$$
N_{2}(y)=\phi(0)+\int_{0}^{t} \int_{0}^{s} v(u) d u d s
$$

In the sequel, let $B_{q}=\{y \in \Omega:\|y\| \leq q\}$.

Step $1 . N_{1}$ is a contraction.

Let $y, \bar{y} \in B_{q}$. By the assumption, we have

$$
\begin{aligned}
\left\|N_{1}(y)-N_{1}(\bar{y})\right\| \leq & \int_{0}^{t}\left\|g\left(s, y_{s}\right)-g\left(s, \bar{y}_{s}\right)\right\| d s+\sum_{0<t_{k}<t}\left\|I_{k}\left(y\left(t_{k}\right)\right)-I_{k}\left(\bar{y}\left(t_{k}\right)\right)\right\| \\
& +\sum_{0<t_{k}<t}\left(T-t_{k}\right)\left\|\bar{I}_{k}\left(y\left(t_{k}\right)\right)-\bar{I}_{k}\left(\bar{y}\left(t_{k}\right)\right)\right\| \\
\leq & p \int_{0}^{t}\left\|y_{s}-\bar{y}_{s}\right\| d s+\sum_{k=1}^{m} c_{k}\left\|y\left(t_{k}\right)-\bar{y}\left(t_{k}\right)\right\| \\
& +\sum_{k=1}^{m}\left(T-t_{k}\right) d_{k}\left\|y\left(t_{k}\right)-\bar{y}\left(t_{k}\right)\right\| \\
\leq & {\left[p T+\sum_{k=1}^{m}\left(c_{k}+\left(T-t_{k}\right) d_{k}\right)\right]\|y-\bar{y}\|_{\Omega} . }
\end{aligned}
$$

Then

$$
\left\|N_{1}(y)-N_{1}(\bar{y})\right\|_{\Omega} \leq\left[p T+\sum_{k=1}^{m}\left(c_{k}+\left(T-t_{k}\right) d_{k}\right)\right]\|y-\bar{y}\|_{\Omega} .
$$

From (3.1), we see that $N_{1}$ is a contraction.

Step 2. $N_{2}(y)$ is convex for each $y \in \Omega$.

Indeed, if $h_{1}, h_{2} \in N_{2}(y)$, then there exist $v_{1}, v_{2} \in S_{F, y}$ such that

$$
h_{i}(t)=\phi(0)+\int_{0}^{t} \int_{0}^{s} v_{i}(u) d u d s, \quad i=1,2, t \in J .
$$


Let $\lambda \in[0,1]$. Then, for each $t \in J$, we have

$$
\left(\lambda h_{1}+(1-\lambda) h_{2}\right)(t)=\phi(0)+\int_{0}^{t} \int_{0}^{s}\left[\lambda v_{1}(u)+(1-\lambda) v_{2}(u)\right] d u d s
$$

Since $S_{F, y}$ is convex (because $F$ has convex values), $\lambda h_{1}+(1-\lambda) h_{2} \in N_{2}(y)$.

Step 3. $N_{2}$ maps bounded sets into bounded sets in $\Omega$.

Indeed, it is enough to show that there exists a positive constant $\Lambda$ such that for each $h \in N_{2}(y), y \in B_{q}=\{y \in \Omega:\|y\| \leq q\}$, one has $\|h\| \leq \Lambda$. If $h \in N_{2}(y)$, then there exists $v \in S_{F, y}$ such that for each $t \in J$, we have

$$
h(t)=\phi(0)+\int_{0}^{t} \int_{0}^{s} v(u) d u d s
$$

By the assumption, for each $t \in J$, we have $\|h(t)\| \leq\|\phi\|_{\mathcal{D}}+\int_{0}^{T} \int_{0}^{s} \varphi_{q} d u d s:=\Lambda$. Then, for each $h \in N_{2}(y)$, we have $\|h(t)\| \leq \Lambda$.

Step 4. $N_{2}$ maps bounded sets into equicontinuous sets of $\Omega$.

Let $0<\tau_{1}<\tau_{2} \leq T$. Then we have, for each and $y \in B_{q}$ and $h \in N_{2}(y)$, the existence of $v \in S_{F, y}$ such that (3.2) hold. Therefore

$$
\left\|h\left(\tau_{2}\right)-h\left(\tau_{1}\right)\right\| \leq \int_{\tau_{1}}^{\tau_{2}} \int_{0}^{s} v(u) d u d s
$$

The right-hand side of the above inequality tends to zero as $\tau_{2} \rightarrow \tau_{1}$. Thus, the set $\left\{N_{2} y\right.$ : $\left.y \in B_{q}\right\}$ is equicontinuous.

Step 5. $N_{2} y$ is a compact multivalued map.

From the above claims, we see that $N_{2} B_{q}$ is a uniformly bounded and equicontinuous collection. Therefore, it suffices to show by the Arzelá-Ascoli theorem that $N_{2}$ maps $B_{q}$ into a precompact set into $\Omega$. That is, for each fixed $t \in J$, the set $V(t)=\left\{h(t): h \in B_{q}\right\}$ is precompact in $E$.

Obviously, $V(0)=\{N(0)\}$. Let $t>0$ be fixed and for $0<\epsilon<t$, define

$$
h_{\epsilon}(t)=\phi(0)+\int_{0}^{t-\epsilon} \int_{0}^{s} v(u) d u d s
$$

Since $v \in S_{F, y}$, the set $V_{\epsilon}(t)=\left\{h_{\epsilon}(t): h \in N_{2}\left(B_{q}\right)\right\}$ is precompact in $\Omega$ for every $\epsilon, 0<\epsilon<t$. Moreover, for every $h \in N_{2}\left(B_{q}\right)$,

$$
\left\|h(t)-h_{\epsilon}(t)\right\| \leq\left\|\int_{t-\epsilon}^{t} \int_{0}^{s} v(u) d u d s\right\| \leq T \psi(q) \int_{t-\epsilon}^{t} p_{1}(s) d s .
$$

Therefore, $\left\|h(t)-h_{\epsilon}(t)\right\| \rightarrow 0$, as $\epsilon \rightarrow 0^{+}$, and there are precompact sets arbitrary close to the set $\left\{h(t): y \in N_{2}\left(B_{q}\right)\right\}$. Hence, the Arzelá-Ascoli theorem shows that $N_{2}$ is a compact multivalued map.

Step 6. $N_{2}$ has a closed graph.

Let $y_{n} \rightarrow y_{*}, h_{n} \in N_{2}\left(y_{n}\right)$, and $h_{n} \rightarrow h_{*}$. We will prove that $h_{*} \in N_{2} y_{*}$. Indeed, $h_{n} \in N_{2}\left(y_{n}\right)$ means that there exists $v_{n} \in S_{F, y_{n}}$ such that

$$
h_{n}(t)=\phi(0)+\int_{0}^{t} \int_{0}^{s} v_{n}(u) d u d s, \quad t \in J
$$


We must prove that there exists $v_{*} \in S_{F, y_{*}}$ such that $h_{*}(t)=\phi(0)+\int_{0}^{t} \int_{0}^{s} v_{*}(u) d u d s$, $t \in J$.

We have $\left\|\left[h_{n}(t)-\phi(0)\right]-\left[h_{*}(t)-\phi(0)\right]\right\|_{\Omega} \rightarrow 0$, as $n \rightarrow \infty$. Consider the linear continuous operator $\Gamma: L^{1}(J, E) \rightarrow C(J, E), v \mapsto \Gamma(v)(t)=\int_{0}^{t} \int_{0}^{s} v(u) d u d s$. From Lemma 2.1, it follows that $\Gamma \circ S_{F}$ is a closed graph operator. Moreover, we have $\left(h_{n}(t)-\phi(0)\right) \in \Gamma\left(S_{F, y_{n}}\right)$.

Since $y_{n} \rightarrow y_{*}$, it follows from Lemma 2.1 that $\left(h_{*}(t)-\phi(0)\right) \in \Gamma\left(S_{F, y_{*}}\right)$, that is, there must exist a $v_{*} \in S_{F, y_{*}}$, such that

$$
h_{*}(t)-\phi(0)=\int_{0}^{t} \int_{0}^{s} v_{*}(u) d u d s .
$$

Therefore, $N_{2}$ is a completely continuous multivalued map, u.s.c. with convex closed, compact values.

Step 7. A priori estimate.

Now it remains to show that the set $G=\left\{y \in \Omega: y \in \lambda N_{1} y+\lambda N_{2} y\right.$ for some $\left.0<\lambda<1\right\}$ is bounded. Let $y \in G$, then there exists $v \in S_{F, y}$ such that

$$
\begin{aligned}
y(t)= & \lambda \phi(0)+\lambda[\eta-g(0, \phi(0))] t+\lambda \int_{0}^{t} g\left(s, y_{s}\right) d s+\lambda \int_{0}^{t} \int_{0}^{s} v(u) d u d s \\
& +\lambda \sum_{0<t_{k}<t}\left[I_{k}\left(y\left(t_{k}\right)\right)+\left(t-t_{k}\right) \bar{I}_{k}\left(y\left(t_{k}\right)\right)\right]
\end{aligned}
$$

for some $0<\lambda<1$. Then, by the assumptions, we have

$$
\begin{aligned}
\|y(t)\| \leq & \|\phi\|_{\mathcal{D}}+T\left(|\eta|+c_{1}^{*}\|\phi\|_{\mathcal{D}}+2 c_{2}^{*}\right)+\int_{0}^{t} c_{1}^{*}\left\|y_{s}\right\|_{\mathcal{D}} d s \\
& +\int_{0}^{t} p_{1}(s) \int_{0}^{s} \psi\left(\left\|y_{u}\right\|_{\mathcal{D}}\right) d u d s+\sum_{k=1}^{m}\left[c_{k}^{\prime}+\left(T-t_{k}\right) d_{k}^{\prime}\right] .
\end{aligned}
$$

We consider the function $\mu$ defined by $\mu(t):=\sup \{|y(s)|:-r \leq s \leq t\}, 0 \leq t \leq T$. Let $t^{*} \in[-r, t]$ be such that $\mu(t)=\left|y\left(t^{*}\right)\right|$.

If $t^{*} \in J$, by the above inequality, we have, for $t \in[0, T]$,

$$
\begin{aligned}
\mu(t) \leq & \|\phi\|_{\mathcal{D}}+T\left(|\eta|+c_{1}^{*}\|\phi\|_{\mathcal{D}}+2 c_{2}^{*}\right)+\int_{0}^{t} M(s) \mu(s) d s \\
& +\int_{0}^{t} M(s) \int_{0}^{s} \psi(\mu(u)) d u d s+\sum_{k=1}^{m}\left[c_{k}^{\prime}+\left(T-t_{k}\right) d_{k}^{\prime}\right] .
\end{aligned}
$$

If $t^{*} \in[-r, 0]$, then $\mu=\|\phi\|_{\mathcal{D}}$ and the above inequality holds.

Let us take the right-hand side of the above inequality as $\theta(t)$. Then we have

$$
\begin{aligned}
& \bar{c}=v(0)=\|\phi\|_{\mathcal{D}}+T\left(|\eta|+c_{1}^{*}\|\phi\|_{\mathcal{D}}+2 c_{2}^{*}\right)+\sum_{k=1}^{m}\left[c_{k}^{\prime}+\left(T-t_{k}\right) d_{k}^{\prime}\right], \quad \mu(t) \leq \theta(t), \\
& \theta^{\prime}(t)=M(t) \mu(t)+M(t) \int_{0}^{t} \psi(\mu(s)) d s, \quad t \in[0, T] .
\end{aligned}
$$

Using the nondecreasing character of $\psi$, we get $\theta^{\prime}(t) \leq M(t)\left[\theta(t)+\int_{0}^{t} \psi(\theta(s)) d s\right]$. Put $w(t)=\theta(t)+\int_{0}^{t} \psi(\theta(s)) d s, t \in[0, T]$. Then $w(0)=\theta(0), \theta(t) \leq w(t), t \in[0, T]$, and $w^{\prime}(t)=$ 
$\theta^{\prime}(t)+\psi(\theta(t)) \leq M(t)[w(t)+\psi(w(t))], t \in[0, T]$. This inequality implies, for each $t \in[0, T]$,

$$
\int_{w(0)}^{w(t)} \frac{d s}{s+\psi(s)} \leq \int_{0}^{T} M(s) d s<\int_{\bar{c}}^{\infty} \frac{d s}{s+\psi(s)}
$$

This inequality implies that there exists a constant $b$ such that $w(t) \leq b, t \in[0, T]$, and hence $\mu(t) \leq b, t \in[0, T]$. Since for every $t \in[0, T],\left\|y_{t}\right\|_{\mathcal{D}} \leq \mu(t)$, we have $\|y\| \leq$ $\max \left\{\|\phi\|_{\mathcal{D}}, b\right\}$. Here $b$ depends only on $T$ and on the function $p_{1}$ and $\psi$. This indicates that the set $G$ is bounded.

As a consequence of Theorem 2.1, we deduce that $N_{1}+N_{2}$ has a fixed point which is the mild solution of the problem (1.1).

\section{Example}

As an application of Theorem 3.1, we consider second-order impulsive neutral functional differential inclusions of the following form:

$$
\left\{\begin{array}{l}
\frac{\partial^{2}}{\partial t^{2}} v(t, x) \in \frac{\partial}{\partial t} g(t, v(t-h), x)+\left[f_{1}(t, v(t-h), x), f_{2}(t, v(t-h), x)\right], \\
\quad x \in[0, \pi], t \in J, t \neq t_{k}, \\
v\left(t_{k}^{+}, x\right)-v\left(t_{k}^{-}, x\right)=I_{k}\left(v\left(t_{k}^{-}, x\right)\right), \quad k=1, \ldots, m, \\
v^{\prime}\left(t_{k}^{+}, x\right)-v^{\prime}\left(t_{k}^{-}, x\right)=\bar{I}_{k}\left(v\left(t_{k}^{-}, x\right)\right), \quad k=1, \ldots, m, \\
v(t, 0)=v(t, \pi)=0, \quad t \in J, \\
v(t, x)=\phi(t, x), \quad \frac{\partial}{\partial t} v(0, x)=\eta(x), \quad t \in[-\infty, 0), x \in[0, \pi],
\end{array}\right.
$$

where $J=[0, T], k=1, \ldots, m, v\left(t_{k}^{+}, x\right)=\lim _{s \rightarrow 0^{+}} v\left(t_{k}+s, x\right), v\left(t_{k}^{-}, x\right)=\lim _{s \rightarrow 0^{-}} v\left(t_{k}+s, x\right)$, $f_{1}, f_{2}: J \times R \rightarrow R$ are two given functions. We assume that $I_{k}, \bar{I}_{k}$ are all continuous; assume that for each $t \in J, f_{1}(t, \cdot)$ is lower semicontinuous and for each $t \in J, f_{2}(t, \cdot)$ is super semicontinuous. It is clear that $F=\left[f_{1}, f_{1}\right]$ is compact and convex valued, and it is super semicontinuous. Hence, let

$$
\begin{aligned}
& \phi(\theta)(x)=\phi(\theta, x), \quad(\theta, x) \in(-\infty, 0] \times[0, \pi], \quad v(t)(x)=v(t, x), \\
& I_{k}\left(y\left(t_{k}^{-}\right)\right)(x)=I_{k}\left(v\left(t_{k}^{-}, x\right)\right), \quad \bar{I}_{k}\left(y\left(t_{k}^{-}\right)\right)(x)=\bar{I}_{k}\left(v\left(t_{k}^{-}, x\right)\right), \quad x \in[0, \pi], k=1, \ldots, m, \\
& g(t, \phi)(x)=g(t, \phi(\theta, x))
\end{aligned}
$$

and

$$
F(t, \phi)(x)=\left[f_{1}(t, \phi(\theta, x)), f_{2}(t, \phi(\theta, x))\right], \quad-\infty<\theta \leq 0, x \in[0, \pi]
$$

Hence, (4.1) can be rewritten as the abstract form as the system (1.1). We can define $g, f_{1}$, $f_{2}$ to satisfy the assumptions stated in Theorem 3.1. We omit it here. Therefore, the system (4.1) has at least one mild solution on $J$.

The authors declare that they have no competing interests. 


\section{Acknowledgements}

This work is supported by the NNSF of China (No. 11471109, No. 11571088) and a project supported by Scientific Research Fund of Hunan Provincial Education Department (No. 14A098, No. 14A028) and Hunan Provincial Natural Science Foundation of China (14JJ7083).

Received: 14 April 2015 Accepted: 27 September 2015 Published online: 07 October 2015

\section{References}

1. Benchohra, M, Henderson, J, Ntouyas, SK: Impulsive neutral functional differential equations in Banach spaces. Appl. Anal. 80, 353-365 (2001)

2. Li, WS, Chang, YK, Nieto, JJ: Solvability of impulsive neutral evolution differential inclusions with state-dependent delay. Math. Comput. Model. 49, 1920-1927 (2009)

3. Benchohra, M: Initial value problems for first order impulsive differential inclusions in Banach spaces. Nonlinear Oscil. 4(2), 146-154 (2001)

4. $\mathrm{Hu}, \mathrm{J}, \mathrm{Liu}, \mathrm{X}$ : Existence results of impulsive partial neutral integrodifferential inclusions with infinite delay. Nonlinear Anal. 71, e1132-e1138 (2010)

5. Park, JY, Jeong, JU: Existence result for impulsive neutral stochastic functional integro-differential inclusions with infinite delays. Adv. Differ. Equ. 2014, 17 (2014)

6. Ren, Y, Hu, L, Sakthivel, R: Controllability of impulsive neutral stochastic functional differential inclusions with infinite delay. J. Comput. Appl. Math. 23, 2603-2614 (2011)

7. Dhage, BC: Multi-valued mappings and fixed points II. Tamkang J. Math. 37, 27-46 (2006)

8. Benchohra, M, Henderson, J, Ntouyas, SK: Impulsive neutral functional differential inclusions in Banach spaces. Appl. Math. Lett. 15, 917-924 (2002)

9. Fitzpatrick, PM, Petryshyn, WV: Fixed point theorems for multivalued noncompact acyclic mappings. Pac. J. Math. 54(2), 17-23 (1974)

10. Deimling, K: Multivalued Differential Equations. de Gruyter, Berlin (1992)

\section{Submit your manuscript to a SpringerOpen ${ }^{\ominus}$ journal and benefit from:}

- Convenient online submission

- Rigorous peer review

- Immediate publication on acceptance

- Open access: articles freely available online

- High visibility within the field

- Retaining the copyright to your article 